\title{
Salsola kali var. tenuifolia, una peligrosa maleza exótica que esta extendiéndose hacía el Centro de México
}

\author{
por J. Rzedowsky
}

Sección de Ecología Vegetal. Colegio de Postgraduados. Instituto de la Educación Agrícola Superior. Chapingo, México.

Salsola kali L. var. tenuifolia Tausch $(=S$. pestifer A. Nelson $)$ es una planta originaria de las regiones semi-áridas de Rusia, Cercano Oriente y costas del Mediterráneo. Está adaptada a resistir concentraciones elevadas de sales solubles en el suelo, pero como algunas otras halófitas facultativas prospera bien en habitats ruderales y arvenses.

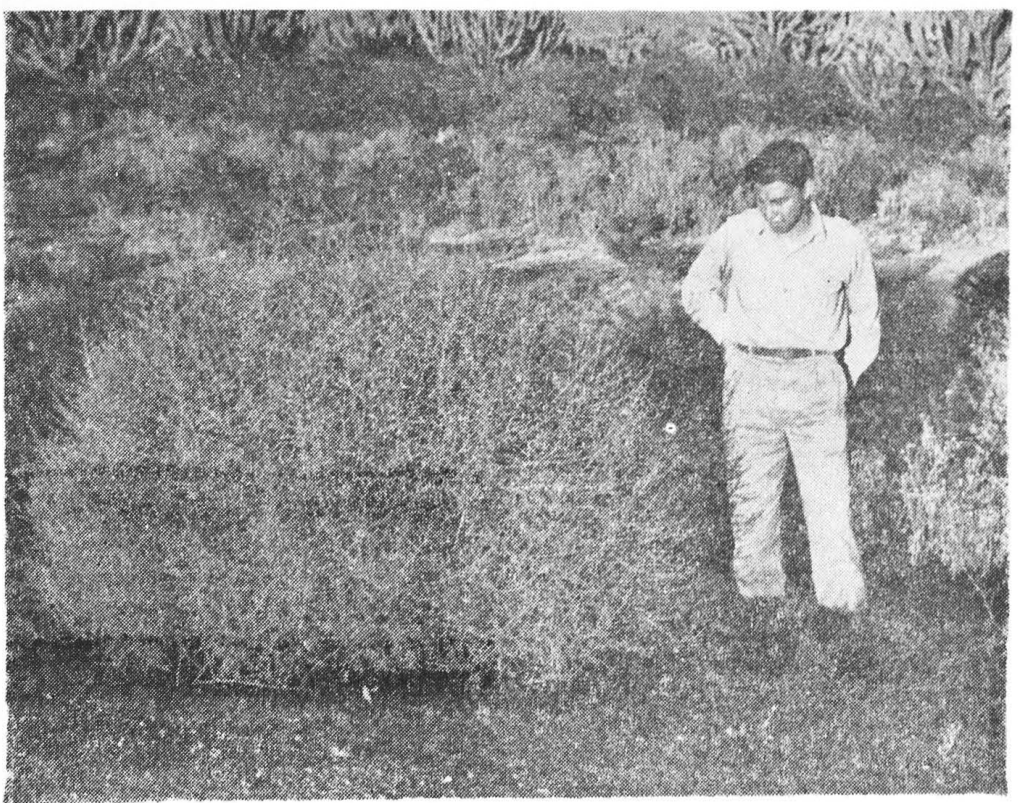

Fig. 1.

Planta de Salsola kali var. tenuifolia en el borde de la carretera central México - Piedras Negras, cerca de Santa Ana Pozas, S. L. P.

Rzedowski J. 1959. Salsola kali var. tenuifolia, una peligrosa maleza exótica que esta extendiéndose hacía el Centro de México. Boletín de la Sociedad Botánica de México 24: 53-59. 
Se trata de un organismo anual que cumple su ciclo vegetativo en la parte caliente del año y es favorecido por la coincidencia de las lluvias en el mismo período. Su crecimiento es rápido y espectacular; se ramifica profusamente y forma masas densas con aspecto de cúpula (fig. 1), que a menudo pasan de $1 \mathrm{~m}$ de alto y $1.5 \mathrm{~m}$ de diámetro. Sus puntiagudas hojas se vuelven espinosas a medida que disminuye su turgencia (fig. 2). Al llegar a la madurez la planta seca se desprende íntegra del substrato, se deja arrastrar sobre la superficie del suelo con ayuda del viento y no suelta las semillas sino en forma paulatina a lo largo de este viaje.

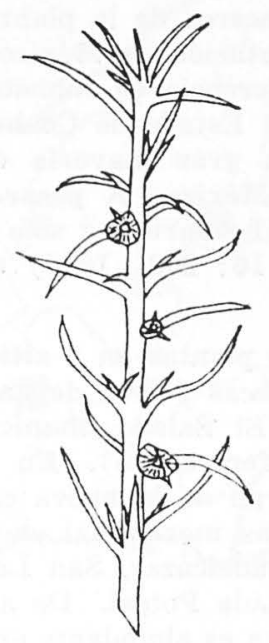

Fig. 2.

Fragmento de rama de Salsola kali var. tenuifolia con frutos inmaduros $(x 1)$

En las zonas meridionales de Rusia Salsola kali var. tenuifolia se comporta como hierba mala en campos de trigo, algodón y otros cultivos (Maltzev, 1931: 301). A los Estados Unidos fue introducida en 1873 (según Dewey, 1895) o en 1887 (según Gilmore, 1932: 67), llegando con semillas de lino que se sembraron en Dakota del Sur. En 1895 su área de distribución abarcaba por lo menos 15 Estados de esa nación, inclusive California, además de algunas porciones de Canadá, Desde esas fechas constituye una de las especies que con mayor frecuencia cubren los bordes de los caminos y los campos abandonados en las partes áridas y semiáridas del Oeste y Medio Oeste americano. Se encuentra en forma esporádica también en la parte oriental de Norteamérica. En ing!és recibe el nombre común de "Russian thistle" (cardo ruso). 
La primera colección de la planta realizada en México fue probablemente la de Endlich, quien la encontró entre Mapimí y Ojuela, Durango, entre 1903 y 1906 (Johnston, 1944: 326). Stearns (1912: 36) la vió en las tierras alcalinas a lo largo del Río Bravo, cerca de Ciudad Juárez, Chihuahua. Gilmore (l. c.) la cita de México sin indicar localidad precisa. Ultimamente, Valdés (1957) la observó en abundancia en campos abandonados cerca de Torreón, Coahuila.

Es muy probable que la penetración de Salsola kali var. tenuifolia a México ha sido lenta y esporádica durante los primeros 50 años del presente siglo. No la cita de este país Standley (1916: 93) y tampoco pudu encontrarse mención alguna acerca de la planta al revisar numerosos trabajos sobre la flora y la vegetación de México aparecidos entre esa fecha y el año de 1957, salvo la referencia de Johnston (l. c.), Ese investigador exploró en forma intensiva el Estado de Coahuila y zonas vecinas, y tuvo la oportunidad de revisar la gran mayoría de las colecciones botánicas provenientes, del Noreste de México. A pesar de ello no encontró ejemplares en el campo ni en los herbarios, y sólo supo a través de la cita de Loesener (Repert. Sp. Nov. 16: 209. 1919) acerca del espécimen encontrado por Endlich.

En 1957 el autor colectó plantas en 2 sitios: terrenos salobres al NE de Concepción del Oro, Zacatecas (cerca de una carretera), y terrenos salobres cerca de la estación El Salado, municipio de Vanegas, San Luis Potosí (cerca de la vía de ferrocarril). En 1959 pudo observar Salsola en diferentes puntos a lo largo de la nueva carretera central México-Piedras Negras; la localidad más meridional encontrada fue cerca de Santa Ana Pozas, municipio de Guadalcázar, San Luis Potosí, a unos $90 \mathrm{~km}$ al NNE de la ciudad de San Luis Potosí. De acuerdo con Miranda (comunicación personal) la planta ya es abundante en los bordes de las carreteras en el Norte del país, particularmente en la Comarca Lagunera. Hernández Xolocotzi localizó desde 1958 una colonia de Salsola sobre suelo salobre del antiguo Lago de Texcoco, en el terreno del poblado de Santa Marta, Distrito Federal, en "frente de la Cárcel de Mujeres, cerca de la ciudad de México. Esta colonia parece estar extendiéndose.

A la luz de los datos anteriores no hay duda de que Salsola kali var. tenuifolia está esparciéndose desde los últimos años en el Norte de México y sus puestos avanzados llegan hasta el Centro del país (fig. 3). Con toda probabilidad un factor importante que ha facilitado su extensión fue la construcción de una red cada vez más densa de carreteras y la creciente circulación de vehículos motorizados. Dada la alta capacidad invasora de la maleza es muy factible que en unos años más se propague a otras zonas de la República, donde su desarrollo no sea incompatible con las condiciones ambientales. En relación con esta circunstancia cabe recordar algunos datos relativos a su ecología, así como señalar posibles implicaciones de orden económico. 


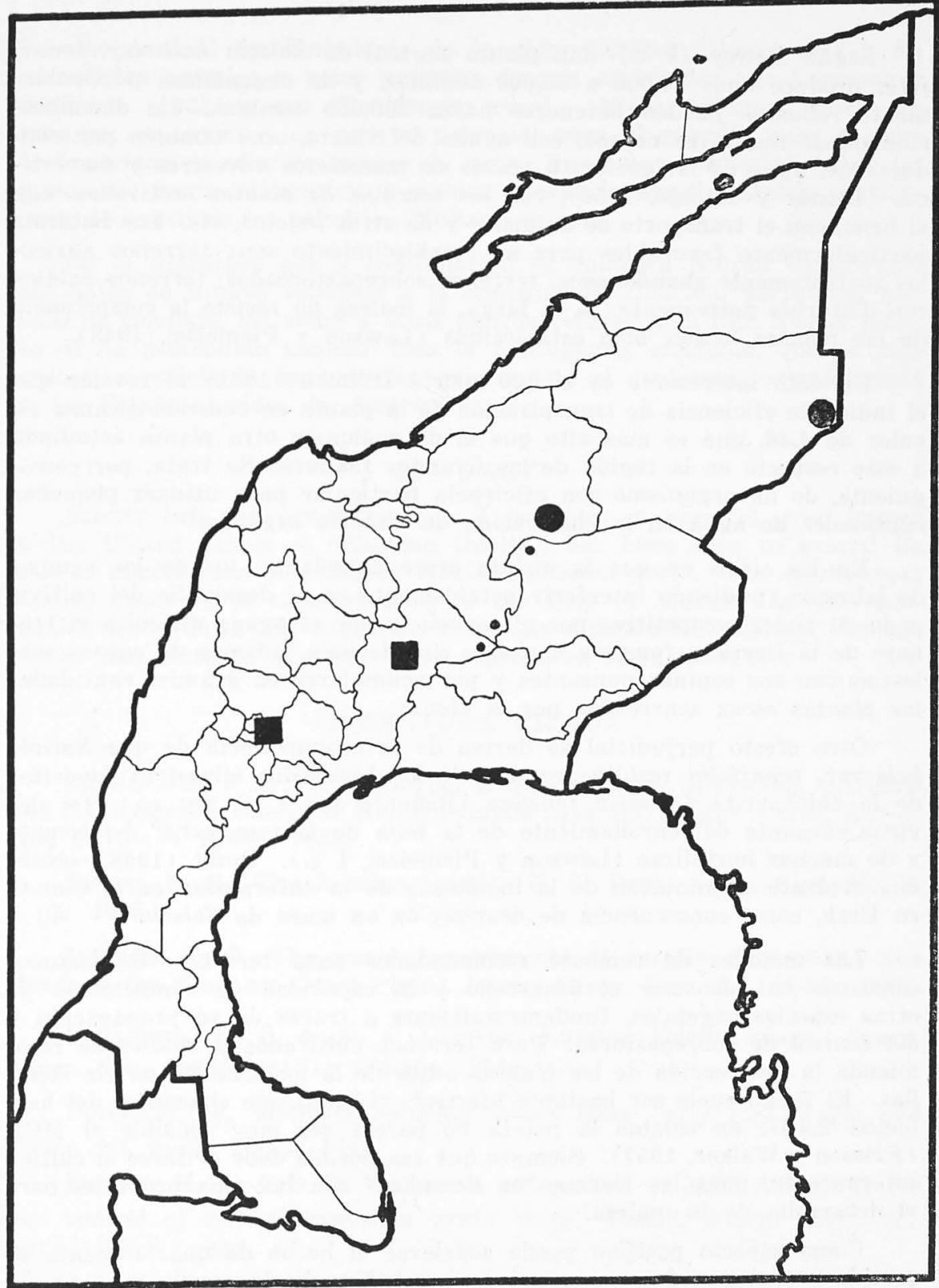

Fig. 3.

Puestos avanzados de Salsola kali var. tenuifolia en México, a principios del siglo (circulos grandes) y en 1959 (cuadrados). Los circulos pequeños señalan otras localidades mencionadas en el texto. 
Según Dewey (1. c.) una planta normal de Salsola kali var. tenuifolia produce unas 20,000 a 30,000 semillas, y de organismos particularmente robustos pueden obtenerse hasta 200,000 semillas. La diseminación puede realizarse no sólo con ayuda del viento, sino también por conducto del agua de irrigación, a través de mamíferos silvestres y domésticos (Lehrer y Tisdale, 1956), con las semillas de plantas cuitivadas, cor el heno, con el transporte de animales y de otros objetos, etc. Los habitats particularmente favorables para su establecimiento son: terrenos agrícolas recientemente abandonados, terrenos sobrepastoreados, terrenos baldíos con disturbio permanente. A la larga, la maleza no resiste la competencia de las plantas locales bien establecidas (Lawson y Piemeisel, 1943).

Un dato interesante es el que aporta Dillman (1931) al revelar que el índice de eficiencia de transpiración de la planta en cuestión alcanza un valor de 4.46, que es más alto que el de cualquier otra planta estudiada a este respecto en la región de las Grandes Llanuras. Se trata, por consiguiente, de un organismo con eficiencia particular para utilizar pequeñas cantidades de agua en la elaboración de materia orgánica.

En los sitios en que la maleza crece abundante, invade los campos de labranza, pudiendo interferir notablemente en el desarrollo del cuitivo, dado su poder competitivo por el espacio y por el agua; dificulta el trabajo de la tierra y fomenta el fuego cuando seca, además de causar molestias con sus espinas punzantes y por acumularse en grandes cantidade: las plantas secas acarreadas por el viento.

Otro efecto perjudicial se deriva de la circunstancia de que Salsola kali var. tenuifolia resulta ser uno de los huéspedes slivestres favoritos de la chicharrita Eutettix tenellus (Baker), que a su vez es vector del virus causante del enrollamiento de la hoja de la remolacha, del tomate y de muchas hortalizas (Lawson y Piemeisel, l. c.). Dorst (1948) señala una evidente disminución de la incidencia de la enfermedad en el tomate en Utah, como consecuencia de destrucc:ón en masa de Salsola.

Los métodos de combate recomendados para terrenos de pastoreo consisten en favorecer el desarrollo y la capacidad de competencia de otras especies vegeta!es, fundamentalmente a través de su propagación y del control de sobrepastoreo. Para terrenos cultivados y baldíos se recomienda la destrucción de las p'antas antes de la maduración de las semillas. El fuego suele ser bastante efectivo, al igual que el empleo del herbicida 2,4-D; en cambio la planta no parece ser muy sensible al MCP (Friesen y Walker, 1957). Siempre que sea posible debe evitarse el cultivo intermitente, pues las tierras "en descanso" son las más propicias para el desarrollo de la maleza.

Como aspecto positivo puede señalarse el hecho de que la planta es comida por el ganado en sus primeros estadíos de desarrollo y se presta a prácticas de ensilaje (Donaldson y Goering, 1940). También puede ser 
de valor para el mantenimiento de la vida silvestre (Long, 1941). Su composición química y valor nutritivo fueron estudiados por Larmour y MacEwan (1938) y por Christensen et al. (1948). Plantas secas, sin embargo, representan un peligro potencial para el ganado, pues a veces son ingeridas en años de escasez de agua. Sus hojas punzantes dañan los tejidos del tracto alimenticio y pueden causar la muerte, siendo particularmente sensibles los caballos (Maltzev, 1. c.).

En la literatura que a continuación se enumera podrán encontrarse más datos de interés relativos a la ecología, distribución, perjuicios, métodos de combate, así como a otros tópicos referentes a esta hierba mala. No se ha pretendido abarcar toda la bibliografía existente, que es muy copiosa y refleja la importancia que reviste el problema, especialmente en los Estados Unidos de América.

\section{SUMMARY}

Salsola kali var. tenuifolia (= S. pestifer), a harmful weed known in the United States as "Russian thistle", has been seen to extend its area of distribution in northern Mexico in recent years. The southermost locality observed is situated close to the City of Mexico. In this connection some important data concerning its distribution, ecology, damage caused and methods of control are reproduced from literature.

\section{LITERATURA CITADA}

Christensen, F. W., T. H. Hopper \& O. A. Stevens, Digestible nutrients and metabolizable energy in Russian-thistle hays and silages. Journ. Agron. Res. 76(3-4): 73-93. 1948.

Dewey, L. H., The Russian thistle. U. S. Dept. Agric. Circ. No. 3. \& pp. 1895.

Dillman, A. C., The water requirements of certain crop plants and weeds in the Northern Great Plains. Journ. Agron. Res. 42: 187-238. 1931.

Donaldson, F. T. \& K. J. Goering, Russian thistle silage. Journ. Amer. Soc. Agron. 32(3): 190-194. 1940.

Dorst, H. E., Methods reducing losses from curly top. Farm and Home Sci. 9(2): 10-12. 1948.

Friesen, H. A. \& D. R. Walker, A comparison of MCP and 2,4-D for the control of annual weeds in grain crops. Canad. Journ. Plant Sci. 37 (1): 69-81. 1957.

Gilmore, M. R., P'ant vagrants in America. Papers Michigan Acad. Sci. Arts Lett. 15. 05-79 1932. 
Johnston, I. M., Plants of Coahuila, e astern Chihuahua, and adjoining Zacatecas and Durango. V. Journ. Arno!d Arbor. 25: 133-182. 1944.

Larmour, R. K. \& J. W. G. MacEwan, The chemical composition of Russian thistle (Salsola pestifer A. Nels.). Sci. Agr. (Ottawa) 18(12): 695-699. 1938.

Lawson, F. R. \& R. L. Piemeisel, The ecology of the principal summer weed hosts of the beet leafhopper in the San Joaguin Valley. California. U. S. Dept. Agric. Techn. Bull. No. 848. 37 pp. 1943.

Lehrer Jr., W. P. \& E. W. Tisdale, Effect of sheep and rabbit digestion on the viability of some range plant seeds. Journ. Range Managm. $9(3)$ : 118-122. 1956.

Long, W. S., The utilization of Russian thistle by wildlife. Journ. Wildlife Managm. 5(2): 136-138. 1941.

Maltzev, A. I., Weeds of the cotton plant. Bull. Appl. Bot. Gen. Plant Breed. 26(5): 287-330. 1931.

Standley; P. C., Chenopodiaceae, in North Amer. Flora 21(1): 1-93. 1916.

Stearns, E., Flora de la región norte de Chihuahua. Secretaría de Fomento. Boletín No 38. 49 pp. México, D. F., 1912.

Valdés, J., Contribución al estudio de la vegetac:ón y de la flora de algunos lugares del norte de México. Tesis. Facultad de Ciencias. Universidad Nacional Autónoma de México. 76 pp. 1957. 